\title{
Sintomas obsessivo-compulsivos na depressão pós-parto: relatos de casos
}

\author{
Obsessive-compulsive symptoms in postpartum depression: case reports
}

\author{
Carla Fonseca Zambaldi', Amaury Cantilino ${ }^{2}$, Everton Botelho Sougey ${ }^{3}$ \\ ${ }^{1}$ Psiquiatra. Assistente do Ambulatório de Depressão Pós-Parto, Universidade Federal de Pernambuco (UFPE), Recife, PE. Mestranda, Pós- \\ Graduação em Neuropsiquiatria e Ciências do Comportamento, UFPE. ${ }^{2}$ Psiquiatra. Coordenador, Programa de Saúde Mental da Mulher, UFPE. \\ Doutorando, Pós-Graduação em Neuropsiquiatria e Ciências do Comportamento, UFPE. ${ }^{3}$ Psiquiatra. Pós-doutor. Professor adjunto, Departamento \\ de Neuropsiquiatria, UFPE. Coordenador, Pós-Graduação em Neuropsiquiatria e Ciências do Comportamento, UFPE.
}

\begin{abstract}
Resumo
A depressão pós-parto é o transtorno afetivo mais prevalente no puerpério. O seu quadro clínico apresenta algumas peculiaridades sintomatológicas, podendo uma delas ser a presença mais freqüente de obsessões e compulsões. Relatamos seis casos identificados pela análise de prontuários de puérperas atendidas no Programa de Saúde Mental da Mulher do Hospital das Clínicas da Universidade Federal de Pernambuco. Todas elas tinham diagnóstico de depressão através do Structured Clinical Interview for DSM-IV Axis $I$ Disorders (SCID-I) e apresentavam concomitantemente sintomas obsessivo-compulsivos. Nos relatos, abordamos o período de aparecimento desses sintomas nas mulheres deprimidas, assim como o seu conteúdo, duração e resposta ao tratamento. Em duas mulheres, os sintomas obsessivo-compulsivos precederam os depressivos, e em outras duas, deu-se o inverso. Houve exacerbação de obsessões e compulsões preexistentes em duas puérperas. O conteúdo mais freqüente foi de pensamentos agressivos contra o bebê. Os sintomas tenderam a diminuir juntamente com a melhora da depressão.
\end{abstract}

Descritores: Depressão pós-parto, puerpério, obsessão, compulsão, comorbidade.

\begin{abstract}
Postpartum depression is the most common affective disorder in the puerperium. There are some particular symptoms in its clinical presentation, and one might be the higher frequency of obsessions and compulsions. We report six cases identified from the analysis of medical charts of puerperal women receiving care at the Women's Mental Health Program, Hospital das Clínicas, Universidade Federal de Pernambuco, Brazil. All the women were diagnosed with postpartum depression using the Structured Clinical Interview for DSM-IV Axis I Disorders (SCID-I) and had associated obsessive-compulsive symptoms. We report time of onset, topics, course and treatment response of these symptoms. Obsessive-compulsive symptoms preceded depressive symptoms in two women, and were succeeded in two other women. There was exacerbation of preexisting obsessions and compulsions in two puerperal women. The most frequent topic was aggressive thoughts toward the baby. Improvement in depressive symptoms tended to reduce obsessivecompulsive symptoms.
\end{abstract}

Keywords: Postpartum depression, puerperium, obsessions, compulsions, comorbidity.

\section{Introdução}

A depressão pós-parto (DPP) é o transtorno afetivo mais freqüente no puerpério, acometendo cerca de $13 \%$ das mulheres nesse período ${ }^{1}$. Em estudos realizados no Brasil, observa-se uma taxa de prevalência que pode variar entre 12 e $37,1 \% \%^{2-5}$. O quadro clínico caracterizase por humor deprimido, desânimo, perda de prazer, cansaço, falta de concentração, alterações de sono e apetite, com início, em geral, em torno de 2 a 3 semanas após o parto ${ }^{6}$.

Tem sido observada, na prática clínica e em pesquisas científicas, a presença de sintomas obsessivocompulsivos, também denominados transtorno obsessivo-compulsivo subclínico em comorbidade com a $\mathrm{DPP}^{7-9}$. Obsessões e compulsões são mais freqüentes 
na DPP que na depressão fora do pós-parto ${ }^{8}$. Os temas mais comuns são pensamentos obsessivos agressivos contra o bebê, imagens envolvendo morte ou algo terrível ocorrendo com o bebê e compulsões de checagem e lavagem ${ }^{9,10}$.

O surgimento de obsessões e compulsões no puerpério parece estar relacionado à queda dos hormônios gonadais e sua influência sobre o sistema serotoninérgico ${ }^{11,12}$, associado à situação de estresse e de aumento de responsabilidade que representa esse período da vida para a mulher ${ }^{13}$.

Neste trabalho, descrevemos seis casos clínicos de DPP com sintomas obsessivo-compulsivos. Foi feita análise retrospectiva de prontuários de pacientes atendidas no período de setembro de 2006 a janeiro de 2008 no Ambulatório de Depressão Pós-Parto do Programa de Saúde Mental da Mulher da Universidade Federal de Pernambuco. Foram identificados casos com diagnóstico de DPP, feito rotineiramente pela Structured Clinical Interview for DSM-IV Axis I Disorders (SCIDI), e que apresentavam, no quadro clínico, obsessões e compulsões significativas.

\section{Descrição dos casos}

\section{Caso 1}

Mulher com 28 anos, casada, ensino médio completo, necessitou transfusão sangüínea, devido a hemorragia, após o parto normal de seu primeiro filho. Após 3 semanas do parto, passou a apresentar pensamentos obsessivos, com preocupação recorrente de ter sido contaminada com vírus HIV na transfusão. A paciente não conseguia suprimir tais pensamentos, apesar de o resultado de exame laboratorial para o vírus ser negativo. Esse sintoma the causava ansiedade e sofrimento intensos.

Dias depois, passou a ter humor depressivo, choro fácil e freqüente, desânimo, falta de prazer. Associado a isso, começou a apresentar pensamentos intrusivos agressivos contra o bebê, como deixá-lo cair; e também a ter medo de contaminá-lo com o vírus ao colocá-lo em seu colo, trocar fraldas ou amamentar, apesar de considerar tais pensamentos irracionais. Temia estar sozinha com o filho e se sentia insegura ao cuidar dele. Procurou tratamento com 10 semanas de pós-parto e foi diagnosticada como tendo DPP com obsessões de temas somáticos e agressivos. Apresentava função tireoidiana e exames bioquímicos sem alterações. Foi iniciada paroxetina $20 \mathrm{mg}$ e, progressivamente, aumentada para $40 \mathrm{mg}$. Houve melhora dos sintomas depressivos e obsessivos após 3 semanas de tratamento. A paciente continuou amamentando e seguiu em acompanhamento com remissão completa.

\section{Caso 2}

Paciente com 42 anos, casada, ensino superior completo, história pregressa de tratamento para transtorno do pânico em 2001. Quatro semanas após o parto cesariana de seu primeiro filho, apresentou humor deprimido, insônia, perda de peso e apetite, desânimo, angústia e sensação de vazio. Apresentava pensamentos agressivos contra o filho, imagens horríveis recorrentes e intrusivas, como bebê morto no berço, se sufocando, caindo no chão. Sentia culpa por achar que não tinha habilidade e segurança para cuidar do filho. Com 6 semanas de pós-parto, foi iniciado tratamento com sertralina e lorazepam. A sertralina foi progressivamente aumentada até $200 \mathrm{mg}$ ao dia, e houve melhora dos sintomas após 4 semanas. Ela interrompeu a amamentação com 4 meses de pós-parto e seguiu o tratamento de manutenção da depressão.

\section{Caso 3}

Mulher de 37 anos, casada, ensino fundamental incompleto, do lar, sem história prévia de transtorno psiquiátrico. Após o parto cesáreo de seu quarto filho, apresentou infecção puerperal, necessitou de internação e uso de antibioticoterapia. Doze semanas após o parto, passou a apresentar pensamento recorrente de que a infecção poderia ter sido evitada se ela tivesse procurado logo a maternidade assim que os sintomas se iniciaram. Paciente reconhecia que tais pensamentos eram excessivos, não conseguia evitar que eles retornassem à sua mente, o que lhe trazia ansiedade e sofrimento. Tinha humor deprimido, insônia, anedonia, choro fácil e freqüente e angústia. Com 24 semanas de parto, foi iniciada paroxetina; como apresentou sonolência, houve troca para fluoxetina, com aumento até $40 \mathrm{mg}$. Teve resposta após 8 semanas de tratamento.

\section{Caso 4}

Adolescente, 16 anos, 2 semanas após o parto de seu primeiro filho, passou a apresentar obsessões agressivas contra o bebê, como impulsos de beliscá-lo e jogá-lo na lata de lixo, além de imagens recorrentes de deixá-lo cair. Tinha sentimento de tristeza, ansiedade, irritabilidade, impaciência, choro fácil e fadigabilidade. Não se dedicava aos cuidados de recém-nascido e não amamentava. Procurou o ambulatório com 12 semanas de pós-parto. Foi iniciada fluoxetina $20 \mathrm{mg}$. A paciente teve melhora parcial e abandonou o tratamento.

\section{Caso 5}

Mulher, 29 anos, separada, ensino médio completo, desempregada. Tinha história prévia de DPP, após o 
parto do primeiro filho há 7 anos, e de dois episódios depressivos fora do período do pós-parto, todos tratados com fluoxetina. Após 3 semanas do parto do terceiro filho, apresentou humor deprimido, cansaço, desânimo, angústia, sentimentos autodepreciativos, perda de apetite e insônia. Apresentava também pensamentos recorrentes agressivos contra o bebê, como jogá-lo na parede e jogar água quente da panela em cima dele. Isso lhe trazia angústia, preocupação, medo de que esses pensamentos realmente acontecessem e culpa. Por isso, passou a se afastar do recém-nascido e ficar temerosa de estar sozinha com ele. Após 7 semanas do parto, procurou atendimento no ambulatório, onde foi identificada DPP com sintomas obsessivo-compulsivos. Utilizou-se paroxetina, e foi necessário o aumento da dose até se atingir $50 \mathrm{mg}$ dia, em associação com cloxazolam. Após 4 semanas, apresentou melhora parcial dos sintomas depressivos e já não tinha sintomas obsessivos.

\section{Caso 6}

Mulher de 40 anos, ensino superior completo, solteira. Primeira gestação foi interrompida com 22 semanas, com parto induzido, devido a feto morto. Cerca de 10 dias depois, relatava se sentir triste e angustiada; chorava muito, tinha dificuldade de concentração, desânimo, anedonia e isolamento social. Paciente já apresentava há alguns anos obsessão por simetria e alinhamento acompanhada de pensamento mágico, que não lhe causava significativas repercussões. Durante esse período, houve uma considerável intensificação dos sintomas. Passou a não caminhar entre poste e muro de uma casa, pois pensava que algo de ruim poderia the acontecer; os objetos de sua casa eram alinhados de maneira a formar a combinação de número três, do contrário pensava que seus planos dariam errado. Sentiase compelida a agir dessa forma, mesmo reconhecendo que tais pensamentos eram excessivos e irracionais. Foi medicada com citalopram $40 \mathrm{mg}$ e encaminhada à psicoterapia. Após 3 semanas de tratamento, apresentou melhora dos sintomas depressivos e diminuição da intensidade e das repercussões dos sintomas obsessivocompulsivos, voltando ao padrão anterior.

\section{Discussão}

Observamos, nesses relatos de caso, que a média de idade foi de 32 anos, que três pacientes eram casadas, duas solteiras e uma divorciada, e que os sintomas depressivos se iniciaram entre 10 dias e 4 semanas após o parto. Em duas pacientes, o início dos sintomas obsessivo-compulsivos precedeu os depressivos; em duas, sucedeu-se o inverso; nas outras duas, houve exacerbação de obsessões e compulsões preexistentes.
Pensamentos agressivos contra o bebê foi o tema mais comum entre as mães deprimidas. Duas pacientes relataram temor de estar com o recém-nascido. Segundo Abramowitz ${ }^{14}$, puérperas que apresentam pensamentos obsessivos agressivos podem considerar-se pessoas perigosas; geralmente temem estar sozinhas com o bebê e evitam estar perto dele, na tentativa de impedir que se concretizem seus pensamentos obsessivos agressivos, o que prejudica a interação mãe-bebê.

Neste estudo, observamos que as mulheres demoraram a procurar o ambulatório de 2 a 12 semanas após o início dos sintomas, provavelmente porque muitas não revelaram seus sintomas de depressão devido a culpa e receio de estigmatização ${ }^{15}$, já que é socialmente esperado delas que estejam felizes com o nascimento do bebê.

Com o tratamento antidepressivo, houve melhora dos sintomas depressivos e dos sintomas obsessivocompulsivos. O tempo médio para resposta ao tratamento foi de 4 semanas. A paciente do caso 3 necessitou 8 semanas para obter melhora de seus sintomas e apresentou o maior período de latência entre o início do quadro e a procura para tratamento. Sabe-se que quanto maior for o atraso entre a instalação da DPP e o início da adequada intervenção farmacológica ou psicológica, maior será a duração do transtorno do humor ${ }^{16}$.

A DPP tem repercussões negativas na qualidade de vida da mulher, na dinâmica familiar, na relação mãebebê e no desenvolvimento cognitivo e afetivo da criança $^{17-20}$. É possível que os sintomas obsessivocompulsivos na DPP agravem essas repercussões e tornem o tratamento mais difícil e demorado, sendo necessárias mais pesquisas nessa área.

\section{Referências}

1. O'Hara MW, Swain AM. Rates and risk of postpartum depression: a meta-analysis. Int Rev Psychiatry. 1996;8:37-54.

2. Cantilino A, Carvalho JA, Maia A, Albuquerque C, Cantilino G, Sougey EB. Translation, validation and cultural aspects of postpartum depression screening scale in Brazilian Portuguese. Transcult Psychiatry. 2007;44:672-84

3. Moraes IGS, Pinheiro RT, Silva RA, Horta BL, Sousa PLR, Faria AD. Prevalência da depressão pós-parto e fatores associados. Rev Saude Publica. 2006;40:65-70.

4. Da-Silva VA, Moraes-Santos AR, Carvalho MS, Martins ML, Teixeira NA. Prenatal and postnatal among low income Brazilian women. Braz J Med Biol Res. 1998;31:799-804.

5. Cruz EBS, Simões GL, Faisal-Cury A. Rastreamento da depressão pós-parto em mulheres atendidas pelo Programa de Saúde da Família. Rev Bras Ginecol Obstet. 2005;27:181-8.

6. Cantilino A, Sougey E. Depressão pós-parto: diagnóstico. Neurobiologia. 2004;67:146-51

7. Abramowitz JS, Schwartz SA, Moore KM, Luenzmann KR. Obsessive-compulsive symptoms in pregnancy and the puerperium: a review of the literature. J Anxiety Disord. 2003;17:461-78.

8. Wisner KL, Peindl KS, Gigliotti T, Hanusa BH. Obsessions and compulsions in women with postpartum depression. J Clin Psychiatry. 1999;60:176-80

9. Jennings KD, Ross S, Popper S, Elmore M. Thoughts of harming 
infants in depressed and nondepressed mothers. J Affect Disord. 1999;54:21-8.

10. Ross LE, McLean LM. Anxiety disorders during pregnancy and the postpartum period: A systematic review. J Clin Psychiatry. 2006;67:1285-98.

11. Labad J, Menchón JM, Alonso P, Segalàs C, Jiménez S, Vallejo J. Female reprodutive cycle and obsessive-compulsive disorder. J Clin Psychiatry. 2005;66:428-35.

12. Uguz F, Akman C, Kaya N, Cilli AS. Postpartum-onset obsessivecompulsive disorder: incidence, clinical feature, and related factors. J Clin Psychiatry. 2007;68:132-8.

13. Fairbrother N, Abramowitz JS. New parenthood as a risk factor for the development of obsessional problems. Behav Res Ther. 2007;45:2155-63.

14. Abramowitz JS, Storch EA, Keeley M, Cordell E. Obsessivecompulsive disorder with comorbid major depression: what is the role cognitive factors? Behav Res Ther. 2007;45:2257-67.
15. Epperson CN. Postpartum major depression: detection and treatment. Am Fam Physician. 1999;59:2247-54, 2259-60.

16. England SJ, Ballard C, George S. Chronicity in postnatal depression. Eur J Psychiatry. 1994;8:93-6.

17. Schwengber DDS, Piccinini CA. O impacto da depressão pós-parto para a interação mãe-bebê. Estud Psicol. 2003;8:403-11.

18. Frizzo GB, Piccinini CA. Interação mãe-bebê em contexto de depressão materna: aspectos teóricos e empíricos. Psicologia em Estudo. 2005;10:47-55.

19. Whiffen VE, Gotlib IH. Comparison of postpartum and nonpostpartum depression: clinical presentation, psychiatric history, and psychosocial functioning. J Consult Clin Psychol. 1993;61:485-94.

20. Herrera E, Reissland N, Shepherd J. Maternal touch and maternal child-directed speech: effects of depressed mood in the postnatal period. J Affect Disord. 2004;81:29-39. 